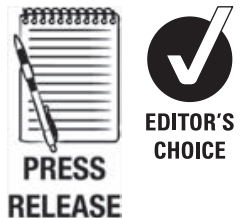

${ }^{1}$ Social Policy Research Unit, University of York, York, UK 2Department of Psychology, University of Stirling, Stirling, UK

\section{Correspondence to} Mairi Harper, Social Policy Research Unit, University of York, York Y010 5DD, UK : mh845@york.ac.uk

Accepted 26 July 2011 Published Online First 1 September 2011

\title{
Increased mortality in parents bereaved in the first year of their child's life
}

Mairi Harper, ${ }^{1}$ Rory C O'Connor, ${ }^{2}$ Ronan E O'Carroll ${ }^{2}$

\section{ABSTRACT}

Objective To identify the relative risk (RR) of mortality in bereaved parents compared with non-bereaved counterparts.

Design Retrospective data linkage study.

Setting United Kingdom, 1971-2006.

Participants A random sample from death registrations (5\%) of parents who had a live birth where the infant lived beyond its first year of life (non-bereaved parents) and parents who had experienced a stillbirth or the death of a child in its first year of life (bereaved parents) between 1971 and 2006.

Main outcome measures Death or widowhood of the parent.

Results Bereaved parents in Scotland $(n=738)$ were more than twice as likely to die in the first 15 years after their child's death than non-bereaved parents $(n=50$ 132), $p<0.005$. Bereaved mothers in England and Wales $(n=481)$ were more than four times as likely to die in the first 15 years after their child's birth than nonbereaved parents $(n=30$ 956), $p<0.001$. The mortality risk for bereaved mothers compared with non-bereaved mothers, followed up for 25 years after death, was 1.5 (bereaved $n=745$, non-bereaved $n=36434$ ), $p<0.005$. When followed up for 35 years, the risk of mortality for bereaved mothers $(n=1120)$ was 1.2 times that of nonbereaved mothers $(n=36062), p<0.005$.

Conclusions Bereaved parents who experience stillbirth or infant death have markedly increased mortality compared with non-bereaved parents, up to 25 years (mean) after the death of their child. However, the $\mathrm{RR}$ reduces over time.

\section{INTRODUCTION}

Mortality following bereavement of a spouse, commonly referred to as "dying of a broken heart", is a well-supported finding. ${ }^{1}$ The situation following the death of one's child is less clear, specifically when the death occurs in infancy, when the highest proportion of child death occurs in the United Kingdom. ${ }^{2}$

For deaths occurring in the first year of a child's life, an increased mortality was found in parents in Denmark compared with the general population of parents. ${ }^{3}$ The risk ratio for the loss of a child aged $<1$ month at time of death was 1.12 (95\% CI 0.83 to 1.52 ), and for a child aged 1-11 months at time of death the risk ratio was 1.29 (95\% CI 0.82 to 2.04). The mortality statistics reported by the age of the child are not broken down into those for mothers and fathers separately. However, results were reported by sex of parent for the death of a child aged up to 18 years. These showed that bereaved mothers have a higher mortality than non-bereaved mothers up to 19 years after the death of their child, but for fathers, increased mortality following bereavement is only found in the first 3 years after loss.

This study establishes, for the first time in the UK, whether bereaved parents who experience the loss of a child in its first year of life have a higher mortality than non-bereaved parents. In part 1 of this study, mortality statistics were compared for a 15-year period, 1991-2006, for both mothers and fathers in Scotland. Part 2 considered the bereavement experience in England and Wales over a 35-year period, between 1971 and 2006. These data are restricted to maternal bereavement only, owing to limitations within the secondary dataset. Differences in mortality between bereaved and non-bereaved parents were compared up to 35 years, to identify if any excess mortality diminished over time. The hypotheses under test were that

(1) Bereaved parents who experience the loss of a child either by stillbirth or in the first year of life would be at higher risk of mortality than non-bereaved parents.

(2) Mortality in bereaved parents would remain higher over time.

\section{METHODS}

Ethical approval for the study was granted by the ethics committee of the Department of Psychology, University of Stirling, and permission to use the Scottish Longitudinal Study (SLS) data was obtained from the Longitudinal Studies Centre, Scotland.

The study comprised two parts. In part 1 , the Longitudinal Studies Centre in Edinburgh, Scotland was contacted to obtain access to data in the SLS database. The SLS is a large-scale linkage study linking together data from administrative and statistical data sources, including census data (1991 and 2001), vital events registrations (to 2008) and NHS Central Register data. The initial SLS sample of 270385 people was drawn from the 1991 census based on a semi-random sample of 20 birthdays, and it represented a 5.3\% sample of the Scottish population. Data for SLS members is linked over time to the 2001 census, and to vital events occurring to them since entering the study. New SLS members enter the study through birth or immigration, and exit the SLS through death or emigration. The subpopulation used in this study was defined as all SLS members who had experienced one or more births, stillbirths or infant mortalities between 1991 and 2001 and who were still present in the 2001 census, plus their partners. If SLS members had had more than one event (ie, live birth and stillbirth or infant 
Table 1 Group characteristics of individuals included in the Scottish analysis

\begin{tabular}{lll}
\hline Characteristics & $\begin{array}{l}\text { Non-bereaved } \\
\text { parents }\end{array}$ & $\begin{array}{l}\text { Bereaved } \\
\text { parents }\end{array}$ \\
\hline $\mathrm{n}$ (SLS members and spouses) & 49828 & 738 \\
Mother: father & $26208: 23620$ & $387: 351$ \\
Mean age in years at birth/death date (SD) & $30.6(5.7)$ & $29.5(6.3)$ \\
Mean years since birth/death date (SD) & $4.7(3.0)$ & $5.7(2.9)$ \\
\hline SLS, Scottish Longitudinal Study. & &
\end{tabular}

mortality), they were coded as having experienced stillbirth or infant mortality events, in preference to live birth events. This approach was taken because the death of the child was the factor which was hypothesised to be associated with increased mortality, thus the death of a child was hypothesised to be the key factor and was therefore given precedence over a live birth experience.

Mortality was measured using death and widowhood status of bereaved SLS members and their spouses. Death and widowhood status were obtained by checking the SLS members' details against the vital events death and widowhood records. At the time of analysis these were available up to 2006. The sample size for the mortality analysis was 28928 SLS members.

The numbers of parents experiencing infant death and stillbirth were supplied already aggregated for death and widowhood data, in order to protect confidentiality owing to the small numbers involved. This group is referred to jointly as "bereaved parents" in this study.

In part 2, data relating to the English and Welsh population from the Office for National Statistics (ONS) Longitudinal Study Dataset was accessed via the Centre for Longitudinal Studies Information and User Support. The ONS dataset contains information from the 1971, 1981, 1991 and 2001 censuses, for people resident in England and Wales, whose date of birth falls on one of four specified dates throughout the year. The database effectively contains data relating to around $1 \%$ of the population of England and Wales (currently around 950 000) study members. Members who were born after 1971 on one of the four dates, or who became resident in England or Wales and had a birthday on one of the key dates, joined the ONS dataset at the first available census. Members are removed from the dataset on death or emigration from England and Wales.

The data available from ONS are similar to those provided by the SLS. Mortality was measured by cross-referencing the ONS database with that of the vital events record in the ONS. Linking information for stillbirths and infant deaths was possible by cross-referencing to the register of births, which is restricted to information only relating to the child's mother. A limitation of the ONS data is, that they, therefore, relate only to the experience of the mother.

At each of the census time points, participants may return results which are incorrectly completed or unreadable. The ONS database marks these as missing data, and these records are therefore excluded for analysis at that particular census point. It is possible, therefore, that members of the ONS dataset may have their data included at one census, be excluded as missing data from the next and then reintroduced at a third time point. Taking this restriction and the methods used for data alignment over time into account, tracking individual members through each of the time points was not feasible within the restrictions of this study. Data are therefore provided for
Table 2 Part one comparison of mortality by group in Scotland

\begin{tabular}{lccr}
\hline & \multicolumn{2}{c}{ Mortality status } & \\
\cline { 2 - 3 } & Alive, N (\%) & Dead, N (\%) & \multicolumn{1}{c}{ Total } \\
\hline Bereaved & $723(97.97)$ & $15(2.03)$ & 738 \\
Non-bereaved & $49650(99.04)$ & $482(0.96)$ & 50132 \\
Total & $50373(99.02)$ & $497(0.98)$ & 50870
\end{tabular}

members at their time of inception into the appropriate cohort, up to 2006 .

To facilitate comparison with the SLS study reported earlier, data from mothers experiencing stillbirth or infant death were aggregated, into one "bereaved parent" group for each census cohort. Again, restrictions on disclosure of information were adhered to, and no data were supplied which could be traced back to potentially identify individual census respondents.

$\chi^{2}$ Tests were carried out on the data using a $2 \times 2$ analysis comparing bereaved parents with non-bereaved parents, with a status of alive/dead.

\section{Role of the funding source}

This study was funded by The Carnegie Trust for the Universities of Scotland. However, the funder had no involvement in the design of the study, data collection or data analyses.

\section{RESULTS: PART 1}

The characteristics of each participant group are shown in table 1.

Ninety seven percent of the population reported an ethnicity status as "white"; $1 \%$ reported ethnicity as "Asian". It was not possible to split these data by bereavement status since this would have been potentially disclosive.

Independent $\chi^{2}$ tests showed that the difference in sex of parent was non-significant. Bereaved parents were slightly younger than the non-bereaved group, $\mathrm{t}(747)=4.6, \mathrm{p}<0.001$ and the mean years since death was slightly higher than the mean years since the birth of a living child, $t(50564)=8.7, p<0.001$ (see table 1 for details). These differences are present owing to the method of establishing birth/death dates for the two groups of participants. If a parent experiences a stillbirth or infant death, this is taken as the birth/death date, regardless of whether a subsequent birth to the parent took place. For live births, the date of the last birth was used as the birth/death date.

Table 2 shows the mortality analysis for both groups. From birth/death date to $2006,482(0.96 \%)$ of the non-bereaved parents died or were widowed, compared with 15 (2.0\%) of the bereaved parents. A $\chi^{2}$ test confirmed that the difference was significant, $\chi^{2}(1)=8.62, p=0.003$ with a risk ratio of $2.11(95 \%$ CI 1.27 to 3.52$)$.

\section{RESULTS: PART 2}

The characteristics of each participant group are shown in table 3 . The table shows the maximum number of census records available. The actual number included in each individual analysis may be less than this total, owing to missing data in subsequent censuses.

Independent $t$ tests showed no significant difference between the age of bereaved and non-bereaved participants in each cohort.

Table 4 shows the results of this mortality analysis for the bereaved and non-bereaved mothers. 
Table 3 Group characteristics of members of each cohort in the English and Welsh analysis

\begin{tabular}{llll}
\hline Characteristics & Cohort & $\begin{array}{l}\text { Non-bereaved } \\
\text { mothers }\end{array}$ & $\begin{array}{l}\text { Bereaved } \\
\text { mothers }\end{array}$ \\
\hline $\mathrm{N}$ & $1971-81$ & 36062 & 1120 \\
$\mathrm{~N}$ & $1981-91$ & 36434 & 745 \\
$\mathrm{~N}$ & $1991-01$ & 30956 & 481 \\
$\begin{array}{l}\text { Mean age at start of census period } \\
\text { in years (SD) }\end{array}$ & $1971-81$ & $22.0(5.7)$ & $21.9(9.0)$ \\
$\begin{array}{l}\text { Mean age at start of census period } \\
\text { in years (SD) }\end{array}$ & $1981-91$ & $22.4(5.7)$ & $22.5(8.4)$ \\
$\begin{array}{l}\text { Mean age at start of census period } \\
\text { in years (SD) }\end{array}$ & $1991-01$ & $24.1(5.2)$ & $23.8(8.3)$ \\
& & &
\end{tabular}

For each cohort, the percentage of bereaved mothers who had died by 2006 was significantly higher than the non-bereaved. A comparison was made between the bereaved and non-bereaved group for each individual cohort. For the 1971-81 cohort, the difference was $\chi^{2}(1)=8.70, p=0.003, n=41797$; RR $=1.24$ (95\% CI 1.08 to 1.42 .) For the 1981-91 cohort, the difference was $\chi^{2}(1)=8.55, p=0.003, n=41208 ; R R=1.50(95 \%$ CI 1.14 to 1.98), and for the 1991-01 cohort, the difference was $\chi^{2}(1)=11.1, p<0.001, n=40631 ; R R=4.74(95 \%$ CI 3.47 to 6.49$)$.

The mean time between the birth/death date and the date of the mother's death was also calculated for each group in each cohort. This was done by subtracting the birth or death date of the child from the date of the maternal death. The results are shown in table 5 .

For the 1971-81 and 1981-91 cohorts, the mean time since death is longer in the bereaved group than in the non-bereaved group. This situation was reversed in the 1991-01 cohorts. The difference was significant for all cohorts. For the 1971-81 cohort, this was $\mathrm{t}(41797)=2.54, \mathrm{p}=0.011, \mathrm{n}=41799$. For the 1981-91 cohort, this was $\mathrm{t}(41206)=2.87, \mathrm{p}=0.004, \mathrm{n}=41208$. Finally, for the 1991-01 cohort, the difference was $t(40629)$ $=14.54, \mathrm{p}<0.001, \mathrm{n}=40631$. Comparison data for individual years was not available since the low numbers involved raised the possibility of potential disclosures for the ONS.

\section{DISCUSSION}

Bereaved parents are between two and four times more likely to die or become widowed in the first 10 years after the experience of stillbirth or the death of their child than non-bereaved parents. Up to 25 years, this risk, for mothers, reduces to 1.5 times that of non-bereaved mothers, and up to 35 years, bereaved mothers still have a higher mortality than the nonbereaved (1.2 times).

This increased mortality in bereaved parents is of major concern. The number of deaths reported herein precludes a more detailed analysis of death by cause. However, largerscale studies should be carried out to identify causes of death and whether there are any identifiable risk factors which might affect this outcome. For example, bereaved parents may be more likely to have committed suicide than matched non-bereaved comparisons. ${ }^{4}$ Insufficient information was provided to rule out the contribution of death by suicide to the difference in mortality statistics. Alternatively, the stress of the bereavement may involve significant physiological effects-for example, suppressing the immune system, ${ }^{5}$ thereby increasing one's propensity to disease. ${ }^{6}$ Bereaved parents may also be more likely to use maladaptive coping strategies such as alcohol misuse. This has been reported by
Table 4 Mortality of cohort members, followed up to 2006, in England and Wales

\begin{tabular}{llrlrrr}
\hline Cohort & Group & n alive & $\%$ & n dead & $\%$ & Total n \\
\hline $1971-81$ & Bereaved & 1094 & 86.0 & 178 & 14.0 & 1272 \\
$1971-81$ & Non-bereaved & 35935 & 88.7 & 4589 & 11.3 & 40524 \\
$1981-91$ & Bereaved & 777 & 94.0 & 50 & 6.0 & 827 \\
$1981-91$ & Non-bereaved & 38758 & 96.0 & 1623 & 4.0 & 40381 \\
$1991-01$ & Bereaved & 622 & 94.0 & 40 & 6.0 & 662 \\
$1991-01$ & Non-bereaved & 39460 & 98.7 & 509 & 1.3 & 39969
\end{tabular}

Table 5 Mean time between birth/death of the child and parental death in individual cohorts, by bereavement status

\begin{tabular}{lll}
\hline Cohort & Status & $\begin{array}{l}\text { Mean time between child birth/death } \\
\text { date and parental death in years (SD) }\end{array}$ \\
\hline $1971-81$ & Bereaved & $20.9(8.7)$ \\
$1971-81$ & Non-bereaved & $20.2(9.7)$ \\
$1981-91$ & Bereaved & $13.6(10.6)$ \\
$1981-91$ & Non-bereaved & $12.7(8.9)$ \\
$1991-01$ & Bereaved & $4.7(5.9)$ \\
$1991-01$ & Non-bereaved & $5.6(1.4)$
\end{tabular}

parents following stillbirth and infant death ${ }^{7}$ and may in turn lead to higher incidence of alcohol-related illness or accidental death. ${ }^{8}$ It is imperative that cause of death be further investigated in order to establish the factors leading to increased mortality in bereaved parents.

Alternatively, it may be that stillbirths and infant deaths are more prevalent in people who, themselves, have serious poorer health, ${ }^{9}$ thus contributing to a lower life expectancy. Further research is required to test these competing explanations for the increased mortality effect.

\section{Limitations of the study}

An important limitation of this study is the restriction of identification of bereaved parents whose child was $<1$ year old at their time of death. This reflects data availability from the secondary datasets used, since parental bereavement is only recorded up to the child's first birthday. More detailed record linkage work could link census and vital events records for parents whose child died at a more advanced age. A comparison group, in this case, would have to be drawn from demographic matching. If it was possible to conduct this further analysis, a rich source of case-controlled information relating to a wide range of parental experiences would provide a clearer view of the mortality risk of parental bereavement.

The data from the ONS related only to bereaved mothers. Although the SLS data indicated that mortality was no different for fathers than for mothers, it is vital that the experience of fathers is considered equally in parental bereavement, and therefore merits further investigation.

In addition, the data supplied are restricted to avoid publishing private and potentially sensitive information. An increased mortality risk of spouses in the first year after bereavement has been noted. ${ }^{10}$ However, the small numbers of bereaved individuals in specific years precluded such a comparison in our study. Additionally, more detailed analyses of specific demographics such as social or ethnic factors and their association with mortality was not possible within the ONS dataset. These more detailed investigations would be facilitated by a larger sample which included a wider range of parental bereavement experiences. 


\section{CONCLUSIONS}

Increased mortality in bereaved parents, initially up to four times those of non-bereaved parents, merits further immediate investigation. Investigations into the causes of death following parental bereavement are required to establish whether specific high-risk groups can be identified, and appropriate interventions delivered.

Acknowledgements The help provided by staff of the Longitudinal Studies Centre - Scotland (LSCS) is acknowledged. The LSCS is supported by the ESRC/JISC, the Scottish Funding Council, the Chief Scientist's Office and the Scottish Executive. The authors are responsible for the interpretation of the data. census output is Crown copyright and is reproduced with the permission of the Controller of HMSO and the Queen's Printer for Scotland. The permission of the Office for National Statistics to use the Longitudinal Study is gratefully acknowledged, as is the help provided by staff of the Centre for Longitudinal Study Information and User Support (CeLSIUS). CeLSIUS is supported by the ESRC Census of Population Programme (Award Ref: RES-348-250004). The authors alone are responsible for the interpretation of the data.

Funding This research was funded under a PhD studentship from the Carnegie Trust for the Universities of Scotland.

\section{Competing interests None.}

Ethics approval The study received ethical approval from the Department of Psychology Ethics Committee within the University of Stirling.

Provenance and peer review Not commissioned; externally peer reviewed.

\section{REFERENCES}

1. Stroebe M, Schut H, Stroebe W. Health outcomes of bereavement. Lancet 2007;370:1960-1973.

2. National Statistics Office. Deaths: age and sex, numbers and rates, 1976 onwards (England and Wales): Population Trends. 2010. http://www.statistics.gov.uk/ STATBASE/ssdataset.asp?vlnk=9552 (accessed 18 August 2011).

3. Li J, Precht DH, Mortensen PB, et al. Mortality in parents after death of a child in Denmark: a nationwide follow-up study. Lancet 2003;361:363-367.

4. Li J, Olsen J. Mortality in parents after death of a child: reply. Lancet 2003;361:1747.

5. $\operatorname{Kim} \mathbf{K}$, Jacobs S. Pathologic grief and its relationship to other psychiatric disorders. J Affect Disord 1991;21:257-263.

6. Vedhara K, Cox NK, Wilcock GK, et al. Chronic stress in elderly carers of dementia patients and antibody response to influenza vaccination. Lancet 1999:353:627-631.

7. Vance JC, Najman JM, Boyle FM, et al. Alcohol and drug usage in parents soon after stillbirth, neonatal death or SIDS. J Paediatr Child Health 1994;30:269-272.

8. Li G, Smith GS, Baker SP. Drinking behavior in relation to cause of death among US adults. Am J Public Health 1994;84:1402-1406.

9. Fretts R. Stillbirth epidemiology, risk factors, and opportunities for stillbirth prevention. Clin Obstet Gynecol 2010;53:588-596.

10. Kaprio J, Koskenvuo M, Rita H. Mortality after bereavement: a prospective study of 95,647 widowed persons. Am J Public Health 1987;77:283-287. 